\title{
THỰC TRANG NĂNG LỰC, NHU CÀU ĐÀO TẠO NGHIÊN CỨU KHOA HOQC CỦA HỌC VIÊN SAU ĐẠI HỌC TẠI ĐẠl HỌC Y HÀ NỌI NĂM 2021
}

\author{
Lê Xuân Hưng ${ }^{\bowtie}$, Nguyễn Thị Thu Hà, Nguyễn Ngọc Anh \\ Viện Đào tạo YHDP và YTCC, Trường Đại học Y Hà Nội
}

Nghiên cứu cắt ngang trên 222 đối tượng học viên sau đại học tại Đại học Y Hà Nội trong đó 59,91\% đã tốt nghiệp Thạc sĩ/CKI và 40,09\% đang học sau Đại học. Điểm trung bình các kỹ năng nghiên cứu của tất cả đối tượng $(3,32 \pm 0,58)$ trên thang đo 5 điểm; Kỹ năng có điểm trung bình thấp nhất là "Phân tích dữ liệu và sử dụng công cụ phân tích" (3,19 $\pm 0,71)$, điểm trung bình cao nhất gồm 2 kỹ năng "Xây dựng đề tài" $(3,34 \pm 0,63)$ và kỹ năng "Lập luận" $(3,34 \pm 0,64)$. Hình thức và phương pháp đào tạo được lựa chọn nhiều nhất "Đào tạo theo nhóm và được hỗ trợ nhiều hơn từ người hướng dẫn" trong nhóm chưa tốt nghiệp (32,58\%) cao hơn so với nhóm đã tốt nghiệp (11,28\%) và phương pháp "Đào tạo lý thuyết song song với thực hành" với nhóm đã tốt nghiệp (56\%) và chưa tốt nghiệp $(62,92 \%)$ sự khác biệt giữa hai nhóm có ý nghĩa thống kê $(p<0,05)$. Tình trạng tốt nghiệp sau đại học và mức độ quan tâm của giảng viên là yếu tố liên quan đến năng lực nghiên cứu của học viên sau đại học.

Từ khóa: Kỹ năng, năng lụ̣c, nghiên cứu khoa học, học viên sau đại học, nhu cầu đào tạo.

\section{I. ĐẠT VẤN ĐÊ}

Quy mô đào tạo sau đại học của nước ta có tốc độ phát triển rất nhanh trong những năm trở lại đây. ${ }^{1}$ Cùng với đó một số trường y khoa ở Việt Nam và trên thế giới đã quy định chuẩn đầu ra cho các học viên sau đại học là các sản phẩm nghiên cứu luận văn, luận án. ${ }^{2}$ Trong đó chất lượng của luận văn, luận án cần phản ánh được chính xác năng lực nghiên cứu mà học viên có khi tốt nghiệp. ${ }^{3}$ Đồng thời, nghiên cứu khoa học ngày càng trở thành kỹ năng quan trọng, cần thiết không chỉ với những học viên khi đang theo học mà cũng là kỹ năng cần thiết trong quá trình công tác sau khi tốt nghiệp trong việc thực hành khoa học dựa trên bằng chứng. Hơn thế luận án tiến sĩ là những công trình khoa học có giá trị với thực tiễn cao, đòi

Tác giả liên hệ: Lê Xuân Hưng

Viện Đào tạo YHDP và YTCC, Trường Đại học Y Hà Nội

Email: lexuanhung@hmu.edu.vn

Ngày nhận: 02/04/2021

Ngày được chấp nhận: 30/07/2021 hỏi nghiên cứu sinh phải có đủ năng lực nghiên cứu khoa học bên cạnh yêu cầu về chuyên môn. ${ }^{4}$ Tuy nhiên, những nghiên cứu trên thế giới và ở Việt Nam cũng đã chỉ ra rằng năng lực nghiên cứu khoa học của các học viên y khoa còn chưa cao, bao gồm những mặt hạn chế cả về kỹ năng xây dựng đề tài, thống kê và cả hoàn thành sản phẩm khoa học. , $^{3,6}$ Nghiên cứu cũng chỉ rõ, thiếu năng lực nghiên cứu khoa học đặc biệt với học viên sau đại học là nguyên nhân chính của những nghiên cứu chất lượng kém hay những nghiên cứu không cần thiết, khó có khả năng đăng tải hoặc công bố thành những sản phẩm khoa học có giá trị. ${ }^{7}$ Bên cạnh đó, các nghiên cứu còn chưa đề cập rõ mức độ tham gia của giảng viên với đề tài nghiên cứu của học viên, đây cũng được xem là một yếu tố quan trọng tác động trực tiếp đến chất lượng sản phẩm và năng lực nghiên cứu khoa học của học viên sau đại học. Việc Trường Đại học Y khoa định hướng là trường đại học nghiên cứu, đòi hỏi cả số lượng và chất lượng của sản 
phẩm nghiên cứu khoa học đều tăng lên và trong đó yếu tố con người đóng một phần quan trọng. Do đó, việc tìm hiểu về "Thực trạng năng lực và nhu cầu nghiên cứu khoa học của học viên sau đại học tại Trường Đại học $Y$ Hà Nội năm 2021" là cần thiết. Nghiên cứu được thực hiện với hai mục tiêu:

1. Mô tả thực trạng năng lực nghiên cứu khoa học của học viên sau đại học tại Đại học $Y$ Hà Nội năm 2021.

2. Xác định nhu cầu đào tạo nghiên cứu khoa học của học viên sau đại học tại Đại học $Y$ Hà Nội năm 2021.

\section{II. ĐÓI TƯƠ'NG VÀ PHƯƠNG PHÁP}

\section{1. Đối tượng}

Học viên sau đại học tại Trường Đại học $Y$ Hà Nội.

\section{Tiêu chuẩn lựa chọn}

- Đã và đang theo học sau đại học tại Trường Đại học Y Hà Nội

- Tự nguyện tham gia nghiên cứu.

\section{Tiêu chuẩn loại trù̀}

- Đối tượng được miễn học khóa học nghiên cứu khoa học.

- Không phải làm luận văn/luận án tốt nghiệp.

\section{Phương pháp}

\section{Thiết kế nghiên cứu}

Mô tả cắt ngang.

\section{Thời gian và địa điểm nghiên cứu}

Từ tháng 9/2020 đến tháng 12/2020 tại trường Đại học $Y$ Hà Nội

\section{Cỡ mẫu và chọn mẫu}

Áp dụng công thức tính cỡ mẫu ước tính một giá trị trung bình trong quần thể theo công thức sau:

$$
n=Z_{1-\alpha / 2}^{2} \frac{s^{2}}{(\bar{X} \cdot \varepsilon)^{2}}
$$

Trong đó:

$Z_{1-\alpha / 2}:$ mức độ chính xác của nghiên cứu cần đạt dự kiến 95\% = 1,96;

s: độ lệch chuẩn thực hiện mô tả tổng kỹ năng nghiên cứu khoa học dựa theo bằng chứng của học viên sau đại học ở Đức $(s=1,08)^{3}$;

$X$ : giá trị trung bình tổng kỹ năng nghiên cứu khoa học $(X=2,88)^{3}$;

$\varepsilon$ : sai số tương đối chấp nhận $(\varepsilon=0,05)$.

Cỡ mẫu tối thiểu là 218. Thực tế thu thập được 222 đối tượng.

\section{Phương pháp chọn mẫu}

Chọn mẫu thuận tiện tập hợp danh sách đối tượng sẵn có gửi qua email lần 1 và được nhắc lại lần 2 sau 1 tuần thu thập.

\section{Biến số nghiên cứru}

Bộ công cụ được xây dựng dựa trên kết quả nghiên cứu của Trần Thanh Ái về các yếu tố tạo nên năng lực nghiên cứu khoa học, ${ }^{8}$ quy trình thực hiện một đề cương nghiên cứu và quy trình học nghiên cứu khoa học sau đại học tại Trường Đại học $Y$ Hà Nội. Bộ công cụ gồm 4 phần: Thông tin chung của đối tượng nghiên cứu , các kỹ năng nghiên cứu khoa học ( trả lời theo thang likert 5 mức độ), nhu cầu đào tạo và các yếu tố liên quan (mức độ tham gia của giảng viên...)

\section{Kỹ thuật và công cụ thu thập số liệu}

Bộ câu hỏi trực tuyến được xây dựng trên công cụ Kobotoolbox và Google drive được gửi cho đối tượng nghiên cứu qua Email đã có sã̃n. Đối tượng nghiên cứu được gửi thư mời tham gia nghiên cứu và thực hiện trả lời bộ câu hỏi. Bộ câu hỏi được xây dựng yêu cầu đối tượng nghiên cứu trả lời đầy đủ các câu hỏi sau đó mới gửi được về cho nhóm nghiên cứu. Những học viên được gửi email để nhắc nhở trả lời bộ câu hỏi theo đúng thời gian thu thập số liệu. Nhóm nghiên cứu kiểm tra câu trả lời những câu trả lời không hợp lệ sẽ được gửi đi và yêu cầu trả lời lại hoặc loại bỏ. 


\section{Xử lý số liệu}

Số liệu sau khi thu thập được tập hợp trên máy chủ. Số liệu sau đó được nghiên cứu viên kiểm tra, làm sạch và xử lý trên phẩm mềm Stata 14.0. Thống kê mô tả tính tần suất, phần trăm, trung bình và mô hình hồi quy logistic được sử dụng để xác định một số yếu tố liên quan giữa biến độc lập (mức độ tham gia của giảng viên, tình trạng học vấn ...) và biến phụ thuộc (điểm trung bình kỹ năng nghiên cứu).

\section{4. Đạo đức nghiên cứu}

Đối tượng nghiên cứu được giải thích rõ mục tiêu nghiên cứu. Nghiên cứu thực hiện trên sự đồng ý tham gia nghiên cứu của đối tượng. Thông tin cá nhân của người trả lời được giữ bí mật và chỉ phục vụ cho mục đích nghiên cứu.

\section{KÉT QUẢ}

1. Thông tin chung về đối tượng nghiên cứu

Bảng 1. Thông tin chung về đối tượng nghiên cứu

\begin{tabular}{lccc}
\hline \multicolumn{2}{c}{ Đặc điểm chung $(\mathbf{n}=\mathbf{2 2 2})$} & Tần số $(\mathbf{n})$ & Tỷ lệ (\%) \\
\hline \multirow{2}{*}{ Giới tính } & Nam & 96 & 43,24 \\
\cline { 2 - 4 } & Nữ & 126 & 56,76 \\
\hline \multirow{2}{*}{ Tốt nghiệp chuyên ngành } & Bác sĩ & 203 & 91,44 \\
\cline { 2 - 4 } & Cử nhân & 19 & 8,56 \\
\hline \multirow{2}{*}{ Tình trạng tốt nghiệp sau Đại học } & Đã tốt nghiệp & 133 & 59,91 \\
\cline { 2 - 4 } & Chưa tốt nghiệp & 89 & 40,09 \\
\hline \multirow{2}{*}{ Đơn vị công tác chính hiện tại } & Bệnh viện & 181 & 81,53 \\
\cline { 2 - 4 } & Khác & 41 & 18,47 \\
\hline
\end{tabular}

Bảng 1 mô tả thông tin chung về đối tượng cho thấy, trong số 222 đối tượng, nữ giới chiếm tỷ lệ cao (56,76\%). 91,44\% đối tượng quan sát là bác sĩ, tiếp đến là cử nhân chiếm ít hơn $(8,56 \%)$. Hơn một nửa trong tổng số đối tượng $(59,91 \%)$ đã tốt nghiệp Thạc sĩ/CKI và 89 đối tượng đang học sau đại học chiếm 40,09\%. Đơn vị công tác chính hiện tại của họ là bệnh viện (81,53\%).

\section{Thực trạng năng lực và nhu cầu nghiên cứu khoa học}

Bảng 2. Điểm trung bình các kỹ năng nghiên cứu khoa học theo tình trạng tốt nghiệp sau Đại học

\begin{tabular}{cccc}
\hline \multirow{2}{*}{ Nội dung } & \multicolumn{3}{c}{ Trung bình (Độ lệch chuẩn) } \\
\cline { 2 - 4 } & $\begin{array}{c}\text { Tổng } \\
(\mathbf{n}=\mathbf{2 2 2})\end{array}$ & $\begin{array}{c}\text { Đã tốt nghiệp } \\
(\mathbf{n}=\mathbf{1 3 3})\end{array}$ & $\begin{array}{c}\text { Chưa tốt nghiệp } \\
\mathbf{( n = 8 9 )}\end{array}$ \\
\hline Xây dựng đề tài & $3,34(0,63)$ & $3,47(0,59)$ & $3,14(0,64)$ \\
\hline Thiết kế nghiên cứu & $3,33(0,61)$ & $3,42(0,6)$ & $3,18(0,6)$ \\
\hline Thu thập thông tin & $3,4(0,65)$ & $3,5(0,69)$ & $3,28(0,59)$ \\
\hline
\end{tabular}




\begin{tabular}{cccc}
\hline \multirow{2}{*}{ Nội dung } & \multicolumn{3}{c}{ Trung bình (Độ lệch chuẩn) } \\
\cline { 2 - 4 } & $\begin{array}{c}\text { Tổng } \\
(\mathbf{n}=\mathbf{2 2 2})\end{array}$ & $\begin{array}{c}\text { Đã tốt nghiệp } \\
(\mathbf{n}=\mathbf{1 3 3 )}\end{array}$ & $\begin{array}{c}\text { Chưa tốt nghiệp } \\
(\mathbf{n}=\mathbf{8 9})\end{array}$ \\
\hline $\begin{array}{c}\text { Phân tích dữ liệu và } \\
\text { sử dụng công cụ phân tích }\end{array}$ & $3,19(0,71)$ & $3,28(0,69)$ & $3,06(0,73)$ \\
\hline Lập luận & $3,34(0,64)$ & $3,47(0,63)$ & $3,12(0,6)$ \\
\hline Tổng các kỹ năng & $3,32(0,58)$ & $3,43(0,58)$ & $3,15(0,55)$ \\
\hline
\end{tabular}

Bảng 2 mô tả điểm trung bình các kỹ năng nghiên cứu khoa học theo tình trạng tốt nghiệp sau đại học theo thang điểm Likert 5 điểm, điểm từ 1 (rất không tốt) đến 5 (rất tốt) cho thấy nhìn chung điểm trung bình tổng các kỹ năng nghiên cứu khoa học của đối tượng nghiên cứu tự đánh giá là cao hơn một chút so với điểm giữa của thang đo là $3(3,32 \pm 0,58)$. Tuy nhiên, trong 2 phân nhóm, điểm trung bình tổng các kỹ năng của nhóm đã tốt nghiệp $(3,43$ $\pm 0,58$ ) cao hơn so với nhóm chưa tốt nghiệp
$(3,15 \pm 0,55)$. Điểm trung bình các kỹ năng của nhóm đã tốt nghiệp đều cao hơn so với nhóm chưa tốt nghiệp.

Kỹ năng có điểm trung bình thấp nhất là "Phân tích dữ liệu và sử dụng công cụ phân tích" $(3,19 \pm 0,71)$ trong đó nhóm đã tốt nghiệp Thạc sĩ/ Chuyên khoa I $(3,28 \pm 0,69)$ và nhóm chưa tốt nghiệp Thạc sĩ/CKI $(3,06 \pm 0,73)$, trong khi đó tổng điểm trung bình cao nhất gồm 2 kỹ năng "Xây dựng đề tài" $(3,34 \pm 0,63)$ và kỹ năng "Lập luận" (3,34 $\pm 0,64)$.

\section{Bảng 3. Nhu cầu đào tạo nghiên cứu khoa học theo năm tốt nghiệp}

\begin{tabular}{lcccccc}
\hline & \multicolumn{3}{c}{ Đã tốt nghiệp } & \multicolumn{2}{c}{ Chưa tốt nghiệp } \\
\cline { 2 - 5 } Đặc điểm nhu cầu đào tạo nghiên cứu khoa học & Tần sốn & Tỷ lện & Tần số & Tỷ lệ & $p$ \\
& $(n)$ & $(\%)$ & (n) & $(\%)$ & \\
\hline
\end{tabular}

\section{Hình thức đào tạo}

\begin{tabular}{llllll}
\hline Giảng dạy theo định hướng nghiên cứu & 74 & 55,64 & 47 & 52,81 & 0,683 \\
\hline $\begin{array}{l}\text { Đào tạo theo nhóm và được hỗ trợ nhiều hơn } \\
\text { từ người hướng dẫn }\end{array}$ & 15 & 11,28 & 29 & 32,58 & $\mathbf{0 , 0 0 0 *}$ \\
\hline $\begin{array}{l}\text { Đào tạo đồng loạt kiến thức cơ bản về nghiên cứu } \\
\text { Phương pháp đào tạo }\end{array}$ & 20 & 15,04 & 22 & 24,72 & 0,082 \\
\hline $\begin{array}{l}\text { Thêm hội thảo chia sẻ về phương pháp nghiên } \\
\text { cứu khoa học }\end{array}$ & 65 & 48,87 & 50 & 56,18 & 0,338 \\
\hline $\begin{array}{l}\text { Tạo môi trường sinh viên tham gia dự án } \\
\text { nghiên cứu có cố vấn }\end{array}$ & 36 & 27,07 & 54 & 60,67 & 0,765 \\
\hline Được giảng viên hướng dẫn và hợp tác nhiều hơn & 41 & 30,83 & 33 & 37,08 & 0,276 \\
\hline
\end{tabular}




\section{Đã tốt nghiệp Chưa tốt nghiệp}

Đặc điểm nhu cầu đào tạo nghiên cứu khoa học $\overline{T a ̂ ̀ n ~ s o ̂ ́ ~} \quad$ Tỷ lệ $\quad$ Tần số $\quad$ Tỷ lệ $\quad p$

(n) $\quad(\%) \quad(n) \quad(\%)$

\begin{tabular}{lccccc}
\hline $\begin{array}{l}\text { Khuyến khích sinh viên thực hiện và xuất bản } \\
\text { bài báo khoa học }\end{array}$ & 34 & 25,56 & 25 & 28,09 & 0,770 \\
\hline $\begin{array}{l}\text { Đào tạo lý thuyết song song với thực hành } \\
\text { Hình thức lượng giá }\end{array}$ & 76 & 57,14 & 56 & 62,92 & $\mathbf{0 , 0 0 0}$ \\
\hline Thực hành một đề tài nghiên cứu theo nhóm & 90 & 67,67 & 61 & 68,54 & 0,384 \\
\hline Thực hiện bài luận cá nhân & 51 & 38,35 & 29 & 32,58 & 0,757 \\
\hline $\begin{array}{l}\text { Thực hiện hoàn chỉnh một nghiên cứu khoa } \\
\text { học cá nhân }\end{array}$ & 17 & 12,78 & 16 & 17,98 & 0,406 \\
\hline Lượng giá trắc nghiệm và sản phẩm thực hành & 39 & 29,32 & 31 & 34,83 & 0,756 \\
\hline
\end{tabular}

${ }^{*}$ Giá trị $p$ ( $p$-value $)<0,05$

Bảng 3 cho thấy nhu cầu đào tạo nghiên cứu khoa học của hai nhóm đối tượng theo năm tốt nghiệp. Về hình thức giảng dạy: Tỷ lệ "Giảng dạy theo định hướng nghiên cứu "của cả hai nhóm đối tượng (đã tốt nghiệp và chưa tốt nghiệp Thạc sĩ/CKI) là cao nhất lần lượt là $(55,64 \%)$ và $(52,81 \%)$. Hình thức "Đào tạo theo nhóm và được hỗ trợ nhiều hơn từ người hướng dẫn" ở nhóm chưa tốt nghiệp (32,58\%) cao hơn so với nhóm đã tốt nghiệp $(11,28 \%)$ và sự khác biệt này có ý nghĩa thống kê $(\mathrm{p}=$ 0,000 ). Đối với phương pháp đào tạo: Tỷ lệ cao

nhất được cả hai nhóm đối tượng đã tốt nghiệp (56\%) và chưa tốt nghiệp $(62,92 \%)$ lựa chọn là phương pháp "Đào tạo lý thuyết song song với thực hành". Tuy nhiên, tỷ lệ lựa chọn phương pháp đào tạo này cao hơn ở nhóm chưa tốt nghiệp so với nhóm đã tốt nghiệp và sự khác biệt có ý nghĩa thống kê $(p=0,000)$. Hình thức lượng giá "Thực hành một đề tài nghiên cứu theo nhóm" được cả hai nhóm đối tượng Đã tốt nghiệp và chưa tốt nghiệp lựa chọn cao nhất chiếm tỷ lệ lần lượt là $67,67 \%$ và $68,54 \%$.

\section{Một số yếu tố liên quan đến thực trạng nghiên cứu khoa học}

Bảng 4. Mức độ tham gia hướng dẫn của giảng viên theo các phần của đề tài nghiên cứu

\begin{tabular}{lcccc}
\hline \multicolumn{1}{c}{ Nội dung } & $\begin{array}{c}\text { Trung bình } \\
\text { (Độ lệch chuẩn) }\end{array}$ & TV (TPV) & Nhỏ nhất & Lớn nhất \\
\hline Tổng quan nghiên cứu khoa học & $3,62(0,77)$ & $3(3-5)$ & 1 & 5 \\
\hline Xác định và lựa chọn vấn đề nghiên cứu & $3,73(0,79)$ & $4(3-4)$ & 1 & 5 \\
\hline Đặt vấn đề & $3,59(0,74)$ & $3(3-4)$ & 1 & 5 \\
\hline Tổng quan tài liệu & $3,58(0,73)$ & $3(3-4)$ & 1 & 5 \\
\hline Phương pháp nghiên cứu & $3,68(0,77)$ & $4(3-4)$ & 1 & 5 \\
\hline Phân tích và xử lý số liệu & $3,56(0,77)$ & $3(3-4)$ & 1 & 5 \\
\hline
\end{tabular}


Nội dung

\begin{tabular}{lllll}
\hline Trình bày kết quả nghiên cứu & $3,63(0,76)$ & $4(3-4)$ & 1 & 5 \\
\hline Bàn luận & $3,63(0,75)$ & $4(3-4)$ & 1 & 5 \\
\hline Kết luận & $3,67(0,78)$ & $3(3-4)$ & 1 & 5 \\
\hline Trình bày tài liệu tham khảo & $3,53(0,73)$ & $4(3-4)$ & 1 & 5 \\
\hline \multicolumn{1}{c}{ Tổng } & $\mathbf{3 , 6 2}(\mathbf{0}, \mathbf{6 7})$ & & \\
\hline
\end{tabular}

Bảng 4 cho thấy tổng điểm trung bình mức độ tham gia vào đề cương nghiên cứu khoa học là $3,62 \pm 0,67$ trên thang điểm 5 . Điểm trung bình mức độ tham gia của giảng viên vào từng phần của đề cương nghiên cứu rất khác nhau, trong đó điểm trung bình mức độ tham gia của giảng viên vào mục "Trình bày tài liệu tham khảo" $(3,53 \pm 0,73)$ và "Phân tích và xử lý số liệu" $(3,56 \pm 0,77)$ được đánh giá thấp nhất và cao nhất là "Xác định và lựa chọn vấn đề nghiên cứu" $(3,73 \pm 0,79)$.

\section{Bảng 5. Một số yếu tố liên quan đến kỹ năng nghiên cứu khoa học}

\begin{tabular}{lccc}
\hline \multicolumn{1}{c}{ Yếu tố } & Hệ số & $\mathbf{P}$ & $\mathbf{9 5 \%}$ KTC \\
\hline Giới (Nam/Nữ) & $-0,149$ & 0,855 & $(-0,176)-0,146$ \\
\hline Ngành học (Bác sĩ/Cử nhân) & 0,142 & 0,316 & $(-0,136)-0,421$ \\
\hline $\begin{array}{l}\text { Tình trạng tốt nghiệp sau Đại học } \\
\text { (Đã tốt nghiệp/Chưa tốt nghiệp) }\end{array}$ & 0,196 & $\mathbf{0 , 0 2 7}$ & $0,22-0,37$ \\
\hline Nơi công tác chính (Bệnh viện/Khác) & $-0,061$ & 0,073 & $(-0,128)-0,005$ \\
\hline Thiếu thời gian thực hành nghiên cứu khoa học & 0,064 & 0,321 & $(-0,63)-0,193$ \\
\hline Thiếu kỹ năng ngoại ngữ & 0,026 & 0,700 & $(-0,197)-0,915$ \\
\hline Thiếu kinh phí thực hiện nghiên cứu & $-0,052$ & 0,472 & $(-0,197)-0,915$ \\
\hline Thiếu tài liệu, giáo trình nghiên cứu khoa học & $-0,05$ & 0,550 & $(-0,218)-0,116$ \\
\hline Thiếu các khóa học đào tạo nghiên cứu khoa học & $-0,007$ & 0,912 & $(-0,141)-0,125$ \\
\hline $\begin{array}{l}\text { Sự tham gia của giảng viên vào từng phần } \\
\text { của đề cương nghiên cứu }\end{array}$ & 0,298 & $\mathbf{0 , 0 0 0 *}$ & $0,168-0,427$ \\
\hline Hệ số chặn & 2,38 & $\mathbf{0 , 0 0 0 ^ { * }}$ & $1,717-3,042$ \\
\hline
\end{tabular}

${ }^{*}$ Giá trị $p(p$-value $)<0,05$

Bảng 5 trình bày ảnh hưởng của một số yếu tố đến điểm trung bình các kỹ năng nghiên cứu khoa học của đối tượng. Phân tích hồi quy đa biến cho thấy các biến như trình độ tốt nghiệp sau đại học và mức độ tham gia của giảng viên đều có ý nghĩa thống kê $(p<0,05)$. Trình độ tốt nghiệp sau đại học $(p=0,027)$ và Sự tham gia của giảng viên vào từng phần của đề cương nghiên cứu" $(p=0,000)$ có mối liên quan dương với điểm trung bình kỹ năng nghiên cứu. Khi 
trình độ tốt nghiệp sau đại học tăng 1 đơn vị thì điểm trung bình kỹ năng tăng 0,196 đơn vị với 95\% KTC $(0,22-0,37)$; sự tham gia của giảng viên vào từng phần của đề cương nghiên cứu tăng 1 đơn vị thì điểm trung bình kỹ năng nghiên cứu tăng 0,289 đơn vị với 95\% KTC $(0,168-0,427)$. Các yếu tố khác như giới tính, thiếu kinh phí nghiên cứu, thiếu kỹ năng ngoại ngữ ... không có ý nghĩa thống kê.

\section{BÀN LUẦN}

Năng lực nghiên cứu khoa học chủ yếu gồm kiến thức, kỹ năng và thái độ. ${ }^{8}$ Năng lực nghiên cứu khoa học đặc biệt với học viên sau đại học có vai trò quan trọng, những hậu quả của việc thiếu năng lực nghiên cứu như xuất hiện những nghiên cứu chất lượng kém gây lãng phí thời gian, công sức, tiền bạc và các nguồn lực khác và nó cũng phi đạo đức. ${ }^{7}$ Một trong những yếu tố quan trọng của năng lực nghiên cứu là kỹ năng có ảnh hưởng đến chất lượng các ấn phẩm khoa học. ${ }^{6}$

Kỹ năng "Phân tích dữ liệu và sử dụng công cụ phân tích" được đối tượng học viên đánh giá có điểm trung bình thấp nhất $(3,19 \pm 0,71)$. Đồng thời, theo nghiên cứu tương tự trên học viên y khoa sau đại học ở Malaysia kỹ năng này cũng được đánh giá không tốt hoặc rất không tốt chiếm tỷ lệ cao trong các kỹ năng nghiên cứu. ${ }^{9}$ Kết quả này có thể lý giải rằng, thống kê bao gồm kỹ năng phân tích xử lý dữ liệu là một môn học khó, do đó việc ứng dụng thống kê vào nghiên cứu là kỹ năng khó. ${ }^{10}$ Kết quả này phù hợp khi nhiều nghiên cứu trên thế giới cũng chỉ ra rằng các lỗi thống kê suy luận cơ bản trong các báo cáo nghiên cứu y sinh học vẫn được nhắc đi nhắc lại, ${ }^{11-13}$ và những lỗi về phân tích và xử lý số liệu cũng được tìm thấy trong những luận văn, luận án. ${ }^{14}$ Mặt khác, đa số đối tượng nghiên cứu chủ yếu là Thạc sĩ và Chuyên khoa II và môn phương pháp nghiên cứu khoa học này các học viên cao học thường học vào năm đầu tiên của khóa học và việc làm luận văn được tiến hành vào năm thứ hai nên khi làm luận văn thì ít nhiều quên các kiến thức đã học. ${ }^{14}$

Kỹ năng được học viên đánh giá với điểm trung bình cao nhất "Xây dựng đề tài nghiên cứu" $(3,34 \pm 0,63)$. Một nghiên cứu của Đức đánh giá cao về kỹ năng xây dựng ý tưởng nghiên cứu của học viên y khoa. ${ }^{3}$ Điều này phù hợp vì những đối tượng tham gia nghiên cứu là đối tượng sau đại học và nhiều hơn ở đối tượng "Đã tốt nghiệp Thạc sĩ và Chuyên khoa I" và hầu hết đều đã thực hiện một sản phẩm khoa học trước đây như luận án, khóa luận ... Mặt khác, kỹ năng xây dựng đề tài nghiên cứu là kỹ năng vô cùng quan trọng và việc giảng dạy môn nghiên cứu khoa học được tập trung vào việc giúp xác định đề tài và mục tiêu nghiên cứu; đồng thời sản phẩm đầu ra khi học môn nghiên cứu khoa học là trình bày đề cương nghiên cứu.

Nhìn chung nhu cầu đào tạo theo hình thức "Giảng dạy theo định hướng nghiên cứu" chiếm tỷ lệ lớn nhất ở cả hai nhóm đối tượng, tuy nhiên tỷ lệ này ở nhóm đã tốt nghiệp Thạc sĩ và Chuyên khoa I $(55,64 \%)$ cao hơn một chút so với Nhóm chưa tốt nghiệp Thạc sĩ/Chuyên khoa I (52,81\%). Kết quả này phù hợp với một nghiên cứu của Thụy Sĩ năm 2017 cũng chỉ ra rằng đa số học viên y khoa sau đại học $(89 \%)$ đều có kế hoạch tiếp tục sự nghiệp theo định hướng nghiên cứu, đặc biệt đối với học viên có nhu cầu theo học lên Tiến sĩ. ${ }^{15}$

Tỷ lệ hình thức "Đào tạo theo nhóm và được hỗ trợ nhiều hơn từ người hướng dẫn" ở nhóm chưa tốt nghiệp $(32,58 \%)$ cao hơn so với nhóm đã tốt nghiệp $(11,28 \%)$ và tỷ lệ nhu cầu đào tạo theo phương pháp "Đào tạo lý thuyết song song với thực hành" ở nhóm chưa tốt nghiệp Thạc sĩ và Chuyên khoa I (62,92\%) cao hơn so với nhóm Đã tốt nghiệp Thạc sĩ và Chuyên khoa I (56\%). Sự khác biệt này có thể là do nhóm đối tượng đã tốt nghiệp Thạc sĩ và Chuyên khoa 
I đã từng bảo vệ luận án tốt nghiệp nên có tự tin thực hiện sản phẩm khoa học một cách độc lập hơn so với nhóm chưa tốt nghiệp Thạc sĩ và Chuyên khoa I. Điều lý giải này phù hợp với một nghiên cứu tương tự tại Đức, nhóm đã tốt nghiệp Tiến sĩ đánh giá khả năng thực hiện nghiên cứu một cách độc lập của họ tự tin hơn so với nhóm chưa tốt nghiệp tiến sĩ. ${ }^{3}$

Tỷ lệ hình thức lượng giá "Thực hành một đề tài nghiên cứu theo nhóm” là cao nhất ở cả 2 nhóm đối tượng. Học tập theo nhóm được đánh giá có tác động đến kết quả học tập của sinh viên y khoa, tạo điều kiện tốt cho việc học và chia sẻ tài liệu. Hơn nữa, tập trung học và làm nghiên cứu theo nhóm có kết quả tích cực khi báo cáo nghiên cứu khoa học. ${ }^{16-19}$

Trình độ tốt nghiệp sau đại học và Sự tham gia của giảng viên vào từng phần của đề cương nghiên cứu là yếu tố liên quan ảnh hưởng đến điểm trung bình kỹ năng nghiên cứu của đối tượng nghiên cứu. Kết quả này tương tự với một số nghiên cứu trên thế giới về yếu tố liên quan đến kỹ năng và năng lực nghiên cứu của học viên và sinh viên y khoa. ${ }^{6,20,21}$

Nghiên cứu của chúng tôi còn một số hạn chế cỡ mẫu chưa đủ lớn, chưa thực hiện bao quát hết tất cả những nhóm học viên sau đại học. Công cụ thu thập online nên việc quản lý đối tượng nghiên cứu còn hạn chế.

\section{KÉT LUẬN}

Điểm trung bình các kỹ năng nghiên cứu khoa học ở cả hai nhóm đối tượng nghiên cứu đều cao hơn một chút so với điểm giữa của thang đo là 3 . Trong đó kỹ năng kém nhất là "Phân tích dữ liệu và sử dụng công cụ phân tích", tuy nhiên nhóm đã tốt nghiệp Thạc sĩ và Chuyên khoa I có điểm trung bình cao hơn so với nhóm chưa tốt nghiệp Thạc sĩ và Chuyên khoa I. Đối tượng nghiên cứu cho thấy sở thích của họ về nhu cầu đào tạo gồm hình thức, phương thức và hình thức lượng giá phù hợp nhất lần lượt là "Giảng dạy theo định hướng nghiên cứu", "Đào tạo lý thuyết song song với thực hành" và "Thực hành một đề tài nghiên cứu theo nhóm". Tình trạng tốt nghiệp sau đại học và mức độ quan tâm của giảng viên là yếu tố liên quan đến năng lực nghiên cứu của học viên sau đại học.

\section{TÀI LIẸU THAM KHẢO}

1. Tạp chí Cảnh sát nhân dân. Thành tựu và một số hạn chế của đào tạo sau đại học ở nước ta Accessed December 7, 2020. http:// csnd.vn/Home/Giao-duc-Dao-tao/408/Thanhtuu-va-mot-so-han-che-cua-dao-taosau-daihoc-o-nuoc-ta.

2. Andrada U, Bolboaca S, Achimas-Cadariu A, Drugan T. Scientific Journal Articles Output from Medical Doctoral Theses. A Comparative Study. Appl Med Inform. 2016;32:81.

3. Epstein N, Huber J, Gartmeier M, Berberat PO, Reimer M, Fischer MR. Investigation on the acquisition of scientific competences during medical studies and the medical doctoral thesis. GMS J Med Educ. 2018;35(2). doi:10.3205/ zma001167.

4. Larivière V. On the shoulders of students? The contribution of $\mathrm{PhD}$ students to the advancement of knowledge. Scientometrics. 2012;90(2):463-481. doi:10.1007/s11192-0110495-6.

5. Goto A, Thi Ngoc Phuong N, Thi Mai Huong $\mathrm{N}$, Hughes J. Building postgraduate capacity in medical and public health research in Vietnam: an in-service training model. Public Health. 2005;119(3):174-183. doi:10.1016/j. puhe.2004.05.005.

6. Ali F, Shet A, Yan W, Al-Maniri A, Atkins S, Lucas $H$. Doctoral level research and training capacity in the social determinants of health at universities and higher education institutions 
in India, China, Oman and Vietnam: a survey of needs. Health Res Policy Syst. 2017;15. doi:10.1186/s12961-017-0225-5.

7. Research - good, bad and unnecessary Testing Treatments - NCBI Bookshelf. Accessed December 7, 2020. https://www.ncbi.nlm.nih. gov/books/NBK66209/\#_ch10_s5_

8. (PDF) Cần làm gì để phát triển năng lực nghiên cứu khoa học giáo dục. ResearchGate. Accessed September 20, 2020. https://www. researchgate.net/publication/260455794_Can_ lam_gi_de_phat_trien_nang_luc_nghien_cuu_ khoa_hoc_giao_duc

9. Loh SY. Research Activity, Skills and Training Needs of Health-Care Professionals Employed in a Leading Medical Centre - A Reflection of its Eroded Professional Autonomy. Ann Womens Health. 2018;2(1):5.

10. García-Berthou E,Alcaraz C. Incongruence between test statistics and $P$ values in medical papers. BMC Med Res Methodol. 2004;4(1):13. doi:10.1186/1471-2288-4-13.

11. VB Nyirongo, MM Mukaka, LV KalilaniPhiri. Statistical Pitfalls in Medical Research. Malawi Med J. 2008; 20(1): 15-18. doi: 10.4314/ mmj.v20i1.10949.

12. Young J. Statistical Errors in Medical Research - a Chronic Disease? Swiss Med Wkly. 2007;137(03-04):41-43. doi: https://doi. org/10.4414/smw.2007.11794.

13. Sara Fernandes-Taylor, Jenny K Hyun, Rachelle N Reeder, et al. Common statistical and research design problems in manuscripts submitted to high-impact medical journals. BMC Res Notes. 2011; 4: 304. doi: 10.1186/17560500-4-304.

14. Hoàng Thị Hải Vân. Thực trạng ứng dụng thống kê trong các luận văn thạc sĩ và bác sĩ nội trú trường Đại học $Y$ Hà Nội và kết quả một số Accessed December 10, 2020. https://sdh.hmu.edu.vn/images/ngcuusinh/ HOANGTHIHAIVANYTCC31.pdf.

15. Kuehnle K, Winkler DT, Meier-Abt PJ. Swiss national MD-PhD-program: an outcome analysis. Swiss Med Wkly. 2009;139(3738):540-546. doi:smw-12790.

16. Woolf K, Potts HWW, Patel S, McManus IC. The hidden medical school: a longitudinal study of how social networks form, and how they relate to academic performance. Med Teach. 2012;34(7):577-586. doi:10.3109/01421 59X.2012.669082.

17. Lovell B. "We are a tight community": social groups and social identity in medical undergraduates. Med Educ. 2015;49(10):10161027. doi:10.1111/medu.12781.

18. Hendry GD, Hyde SJ, Davy P. Independent student study groups. Med Educ. 2005;39(7):672-679. doi:10.1111/j.13652929.2005.02199.x.

19. Boysen PG, Daste L, Northern T. Multigenerational Challenges and the Future of Graduate Medical Education. Ochsner J. 2016;16(1):101-107.

20. Alison JA, Zafiropoulos B, Heard R. Key factors influencing allied health research capacity in a large Australian metropolitan health district. J Multidiscip Healthc. 2017;10:277-291. doi:10.2147/JMDH.S142009.

21. Finch E, Cornwell P, Ward EC, McPhail SM. Factors influencing research engagement: research interest, confidence and experience in an Australian speech-language pathology workforce. BMC Health Serv Res. 2013;13:144. doi:10.1186/1472-6963-13-144. 


\section{Summary \\ RESEARCH COMPETENCIES AND TRAINING NEEDS \\ AMONG POSTGRADUATE STUDENTS AT HANOI MEDICAL UNIVERSITY IN 2021}

This cross-sectional study assessed the research competencies of 222 graduate students from Hanoi Medical University. Of these, 59.91\% graduated with Master or Specialist I degree and $40.09 \%$ pursued graduate studies. Using a questionnaires consisted of questions on a 5 - point scale, the average score of research skills of all participants was $3.32 \pm 0.58$. The skill with the lowest average score is "Data analysis and using analytical tools" (3.19 \pm 0.71$)$, the highest average scores were "Identifying research topic" (3.34 \pm 0.63$)$ and "Developing argument" $(3.34 \pm 0.64)$. Compared to those who had completed graduate studies, those who had not completed graduate studies were significantly more likely to report the need of "group training and more support from supervisors" "Learning theories in parallelwith practice". Graduation status and working with a faculty were factors related to the research quality.

Keywords: Skills, research competencies, scientific research, postgraduate students, training needs. 